\title{
Red Plaque on the Dorsal Hand With Necrosis: Think Before You Amputate
}

\author{
Marc Mir-Bonafé ${ }^{1}$, Javier Aubán-Pariente ${ }^{1}$, Sheila Requena-López ${ }^{1}$, Celia Gómez-de-Castro ${ }^{1}$, \\ Yolanda Hidalgo-García ${ }^{1}$
}

1 Department of Dermatology, Hospital Universitario Central de Asturias, Oviedo, Spain

Key words: cellulitis, neutrophilic dermatosis, neutrophilic dermatosis of the dorsal hands, Sweet syndrome

Citation: Mir-Bonafé M, Aubán-Pariente J, Requena-López S, Gómez-de-Castro C, Hidalgo-García Y. Red plaque on the dorsal hand with necrosis: think before you amputate. Dermatol Pract Concept. 2020;10(4):e2020100. DOI: https://doi.org/10.5826/dpc.1004a100

Accepted: April 19, 2020; Published: October 26, 2020

Copyright: @2020 Mir-Bonafé et al. This is an open-access article distributed under the terms of the Creative Commons Attribution License BY-NC-4.0, which permits unrestricted noncommercial use, distribution, and reproduction in any medium, provided the original author and source are credited.

Funding: None.

Competing interests: The authors have no conflicts of interest to disclose.

Authorship: All authors have contributed significantly to this publication.

Corresponding author: Marc Mir-Bonafé, MD, Department of Dermatology, Hospital Universitario Central de Asturias, Avenida de Roma s/n, Spain. Email: marcmir5@hotmail.com

\section{Case Presentation}

An 82-year-old man presented with fever and an erythematoedematous left index finger. He had a history of myelodysplastic syndrome. He was placed on intravenous antibiotic (amoxicillin/clavulanic acid) due to suspicion of cellulitis. The condition gradually developed blisters and necrosis, and surgeons decided to amputate. In the following days,

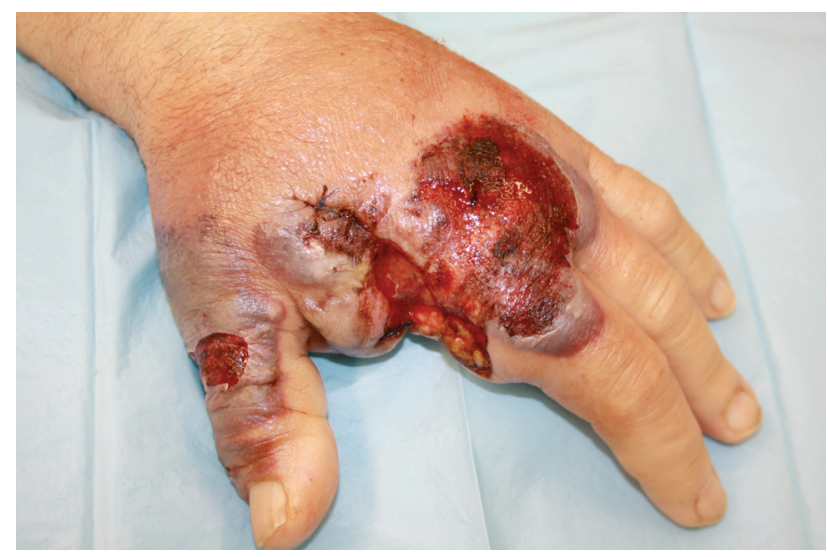

the clinical picture worsened with the development of ulcers with violaceous borders and surrounding edema on the dorsal hand and proximal metacarpophalangeal joints of the thumb and third and fourth fingers (Figure 1). Skin biopsy was compatible with a neutrophilic dermatosis, and the diagnosis of neutrophilic dermatosis of the dorsal hands (NDDH) was made. He was immediately started on oral prednisone $80 \mathrm{mg}$ per day and showed rapid improvement.

\section{Teaching Point}

$\mathrm{NDDH}$, a distributional variant of Sweet syndrome, is an under-recognized entity. It is often clinically misdiagnosed as an infectious process. Physicians should suspect this diagnosis in order to avoid unnecessary and damaging procedures.

Figure 1. Ulcers with violaceous borders and surrounding edema present on the dorsal left hand and aspects of the thumb with proximal metacarpophalangeal joints of the third and fourth fingers. Note the absence of the left index finger. 